\title{
Naming structures and qualifying properties of anuran bioacoustical signals: a call for a homology-based nomenclature and equality for quantitative data
}

\author{
FÁBIO HEPP ${ }^{1}$ and JOSÉ P. POMBAL JR. ${ }^{2}$ \\ ${ }^{1}$ Laboratório de Anfíbios e Répteis, Departamento de Zoologia, Instituto de Biologia, Universidade Federal do Rio de Janeiro, \\ Avenida Brigadeiro Trompowski, s/n, Centro de Ciências da Saúde, Ilha do Fundão, 21941-902 Rio de Janeiro, RJ, Brazil \\ ${ }^{2}$ Departamento de Vertebrados, Museu Nacional, Universidade Federal do Rio de \\ Janeiro, Quinta da Boa Vista, 20940-040 Rio de Janeiro, RJ, Brazil \\ Manuscript received on August 20, 2019; accepted for publication on October 14, 2019
}

\begin{abstract}
How to cite: HEPP F AND POMBAL JP. 2019. Naming structures and qualifying properties of anuran bioacoustical signals: a call for a homology-based nomenclature and equality for quantitative data. An Acad Bras Cienc 91: e20190965. DOI 10.1590/0001-3765201920190965.
\end{abstract}

\begin{abstract}
There is a significant interest for using call features in taxonomic and systematic studies. However, the indiscriminate use of alternative terms for the acoustic structures has precluded reliable comparisons among species without directly checking recordings of all compared species. Recent attempts of nomenclature standardization have focused on term and structure definitions instead of homology. Here, we argue that application of nomenclatural propositions based on universal similarities is pragmatically difficult (likely impossible) and, most importantly, inaccurate regarding to assessment of homology. If homology is not properly assessed, nomenclature can lead to artificial groupings, which can hamper our understanding of the natural world. As a solution, we propose a guideline to first assess homology correspondence and then apply adequate terms to bioacoustical structures. This assessment follows the same homology criteria used to other phenotypical structures, such as morphological ones, and their successful application depend on the comprehension of the acoustic characteristics of the structures and on sampling intermediate forms. Additionally, we point out that there is no biological difference between traits presented qualitatively or quantitatively, and, some of the issues assumedly related to quantitative features are actually related to polymorphism and overlap of trait variation between taxa.
\end{abstract}

Key words: behavior, bioacoustics, comparative biology, systematics, terminology.

Acoustic communication is a very important step in the reproductive cycle of anurans, mediating the encounter of males and females during the mating period (Bogert 1960, Wells 1977, 2007). In this context, males emit calls to advertise their position and individual quality to females, which usually choose the preferred male based on this

Correspondence to: Fábio Hepp

E-mail: fabiohepp@gmail.com

ORCid: https://orcid.org/0000-0001-6100-1275 information (Wells 2007). Moreover, males usually also emit specific calls to other males in order to establish territories and vocalization sites (Wells 1977, 2007). Because acoustic signals are usually species-specific and occur in almost all anuran species, there is a significant interest for using call features in taxonomic and systematic studies on anuran taxa (Köhler et al. 2017). However, the high number of alternative terms available for the same acoustic structures (i.e., homologous structures) in 
literature precludes reliable comparisons among species without directly checking recordings of all compared species (Toledo et al. 2015a). It is long known that one of the main problems related to using anuran bioacoustical traits for taxonomic purposes is the arbitrariness of application of descriptive terms when comparing different studies and authors. The attempts of nomenclature standardization have failed to give universal and effective diagnoses to the bioacoustical structures and then failed to establish a constant and objective terminology over time. Even when clear differentiations between term definitions are achieved, the classification of the structures based on fixed difference limits does not guarantee comparison reliability, since structures of the same class may not be homologous (Ghiselin 2005). Additionally, some authors have tended to prefer qualitative characters than quantitative ones when using bioacoustical features for taxonomic purposes (e.g., Gerhard and Huber 2002, Köhler et al. 2017). As consequence, the comparisons between bioacoustical signals have been made by using only part of the measured traits. Here we aimed to demonstrate that propositions of universal definitions and terms to acoustic structures based on similarity are pragmatically difficult (likely impossible) and, most importantly, inefficient regarding the assessment of homology (Ghiselin 2005). As an alternative, we propose that adequate terms (judged on their definitions) should be applied only after homology correspondence between structures has been made. We also point out that there is no biological difference between traits presented qualitatively or quantitatively, categories which have no natural meaning, instead, they are related only to how researchers decide to describe features. On the other hand, some of the issues assumedly related to quantitative features are actually related to the extension degree of the observed polymorphism and overlap presence of trait variation among taxa.

\section{RIGID DEFINITION FOR BIOACOUSTICAL NOMENCLATURE}

Recently, some studies have presented a list of terms and definitions based on similarity for describing any anuran vocalization in attempt to standardize the nomenclature (e.g., Toledo et al. 2015a, Köhler et al. 2017). The apparent pragmatic advantages from those standardization lists are counterbalanced by the theoretical oversimplification of the diversity while classifying all bioacoustical structures based on rigid and universal definitions. When a large bioacoustical diversity is taken into account, a common situation is a partial match between features of a certain structure and the definition a single term or a similar match with definitions of more than one term. For instance, in order to attenuate the issue, Köhler et al. (2017) presented two alternative approaches to name sound units (structures): one giving priority to the term 'call' (call-centered) and other to the term 'note' (notecentered). Although this flexibility facilitates terminology application, mainly when several species are compared, the practical issues that result from rigid definitions are still present, regardless of which approach is chosen.

The theoretical implications are more serious than the practical difficulties. The fact that some of the purposes of those terminology standardizations are to guide comparisons in further studies, for instance systematics studies, implies that implicit homology propositions have been made (Hall 2013). As a consequence, if homology is not properly assessed, nomenclature can lead to artificial groupings of similar structures, which can hamper our understanding of evolution (see Henning 1966, Grant and Kluge 2004). Although only congruence in phylogenetic analyses serves as a homology test (de Pinna 1991), proper assessment of primary homology is important for character construction (Grant and Kluge 2004, Desutter-Grandcolas et al. 2005) or even for taxonomic comparisons without 
having deep comprehension of the phylogenetic relationships for the studied taxa (e.g., Wenzel 1992, Hall 2013). The most problematic aspect is the risk of using different terms for homologous structures (error I) or using the same term for nonhomologous structures (error II). Both errors are usually related to implicitly equating homology to similarity instead of to correspondence (Ghiselin 2005). Homologous structures are those genealogically connected to a single origin in space and time and they do not need to be similar to each other (Ghiselin 1974, 2005). The establishment of biological parts (= structures) as homologous is supported by finding correspondence among these parts, which does not necessarily mean finding similarity among all of them (Remane 1952, Henning 1966, Ghiselin 2005). If similarity was essential, structures completely different from each other could not be considered homologous even when intermediate forms are present connecting them in a single and gradual transformation series (Grant and Kluge 2004, Ghiselin 2005).

Nevertheless, it should be noticed that similarity is operationally useful while making correspondence among parts (see de Pinna 1991, Desutter-Grandcolas et al. 2005). Current criteria used to recognize correspondence among phenotypical structures are based on similarity between the closest taxa within the transformation series or gradient (Remane 1952, Wenzel 1992; see next section). Still, trait similarities used to support homology correspondences should not be interpreted as fixed and universal evidence but as momentary and particular evidence for connection of the studied taxa. For instance, the same similarities used once to establish homology might be posteriorly reinterpreted as convergences (sensu Desutter-Grandcolas et al. 2005) and then considered not adequate to recognize homologous structures in a particular taxon.

The freedom to reestablish primary homology at any time based on available evidence is crucial.
Structural definitions are inevitably proposed based on a limited number of traits and taxa and the incentive to use them as universal rules to define and classify structures can lead to spurious homology assessments (see convergent structures in Ghiselin 2005). This concern was clearly stated by Köhler et al. (2017: 25): 'Either definition might be appropriate when looking at a single species, but in a comparative taxonomic study, it is of utmost importance to compare homologous bioacoustical entities and to apply the same name to them' (see also the box 'terminology' in Köhler et al. 2017:103). When the correspondences among structures or parts are respected, any universal definition tends to fail, mainly when increasing taxonomic sampling (Rendall and Di Fiore 2007). Every attempt of nomenclature standardization has confronted this problem. For instance, in Köhler et al. (2017:7) the term 'call' was defined as an acoustic unit typically separated from each other by periods of silence much longer than its own duration. In their Fig. 7B, the major unit was indeed named call 'because the silent intervals between them [notes] are much shorter than the units themselves [notes]', agreeing with the call definition aforementioned (Köhler et al. 2017: 6-9). However, in Fig. 7D, the authors stated that there are two calls because they are separated by a long silent interval even with this inter-call interval shorter than the duration of the calls. Actually, in this case, inter-call interval seems to have a duration value similar to that of the inter-note intervals (Köhler et al. 2017: 24). For other cases, claimed to be less subjective (e.g., 'pulse', which was considered 'rather clearly defined'; Köhler et al. 2017: 25), terms received considerably loose definitions. The structure pulse was defined as being 'often [...] not separated by a fully silent interval due to intrinsic properties of the call [...]' [emphasis added]. On the other hand, they stated: '[...] but in some cases, spaced pulses with silent intervals do occur' [emphasis added] (see also Figure 8 of that study; Köhler et al. 2017: 
24). Therefore, short emissions either separated or not by a fully silent interval can be called pulses. Words such as 'often' and 'typically' are present in almost all the structure definitions.

Both flexibilization of term definitions and purposeful misapplication of rigid definitions exemplify attempts to avoid errors I and/or II. Here, we claim that definitions of terms should not interfere in the process of making correspondence among structures (i.e., hypotheses of primary homology). Instead, structure terms and definitions should be discussed and updated only after the assessment of homology (see section 'Naming bioacoustical structures' below). In other words, in order to make homonymous acoustic structures comparable, the homology hypotheses must be the basis of the terminological definitions and not the contrary.

\section{FUNCTION AND PHYSIOLOGICAL FEATURES AS QUALITIES}

A function-based classification is one way to categorize and name anuran calls and their parts (e.g., advertisement call, aggressive note, territorial call, etc.). This approach is the most frequent in literature (e.g., Bogert 1960, Wells 1977, 2007, Duellman and Trueb 1986) and consequently calls are compared if they have the same or similar functions (Toledo et al. 2015a). Although it is expected that homologous calls have the same function, it is important to highlight that homologous calls may not have even similar functions. Function, like any other property, is not static and it can change throughout the evolutionary history of any taxon (Striedter and Northcutt 1991, Rendall and Di Fiore 2007). The function of a signal is only one feature, or a set of features, that can be used to infer homology correspondence (e.g., Robillard and Desutter-Grandcolas 2011; see Striedter and Northcutt 1991, Wenzel 1992, Hall 1994, 2013). Therefore, functional properties described by behavioral observation in field or by playback experiments should not be considered as the ultimate evidence of homology. Instead, the possibility of establishing calls with different functions (e.g., aggressive, courtship, distress) as homologous must be considered. Any feature related to the signal function within the acoustic communication should be interpreted as a 'quality' as any other feature used to assess homology (see Remane's criterion I below).

Recently, it has been claimed that a so called 'mechanistic criterion' would be more objective and efficient to adequately recognize bioacoustical homology in anurans (Robillard et al. 2006, Köhler et al. 2017). Under this criterion, acoustic signals of different species emitted during a single airflow are considered homologous (McLister et al. 1995, Robillard et al. 2006). Similarly, it is often required similar morphological or physiological features underlying similar behavioral expressions in order to consider these expressions homologous (see Rendall and Di Fiore 2007 for a review). Nonetheless, the arguments that once supported the use of such dependency between different character sources as a pillar for behavioral homology have been strongly criticized (Striedter and Northcutt 1991, Hall 1994, 2013, Rendall and Di Fiore 2007). Here, as well as for functions, we suggest that physiologic similarities between behaviors, such as direction (inspiration or expiration) and number of airflows used to produce the signal, are considered quality similarities as any other and they should be taken into consideration as additional independent evidence for homology assessments (see Remane's criterion I below).

\section{QUANTITATIVE VS. QUALITATIVE TRAITS}

A frequent assumption of bioacousticians is that there is difference in reliability between quantitative and qualitative characters for taxonomic comparisons (Gerhard and Huber 2002, Köhler et 
al. 2017). According to Köhler et al. (2017:44 and 50) qualitative characters are more reliable than quantitative ones for taxonomic purposes (see also Gerhard and Huber 2002: 405). The point here is related to the artificiality of these categories. The terms 'qualitative' and 'quantitative' refer only to how features were described at a particular moment and they do not correspond to natural categories (see Thiele 1993, review in Wiens 2001). In other words, any feature can be described either qualitatively or quantitatively (Wiens 2000). Köhler et al. (2017: 46) gave the example of short vs. long sounds to illustrate reliable qualitative differences in contrast to common quantitative traits such as call-structure durations in seconds. However, their example is a very good example to illustrate how the same property can be described qualitatively or quantitatively. In that case, the words 'short' and 'long' were used to refer qualitatively to the relative length or duration of structures, which are usually measured and described numerically (i.e., quantitatively).

Even a typical character such as presence vs. absence of a particular structure (e.g., presence vs. absence of pulses), could be quantitatively described. Absence and presence of structures can be alternatively described by how many of them are present (for well-delimited structures) or by their absolute length (for gradual variation), with integer numbers (= meristic character) or decimal numbers (= continuous character), respectively. For both cases, the absence would be described by the number 0 (zero). The alternative conversion is equally possible, i.e., numerical characters classified into categories according to their relative ranges of variation; for instance, characters with qualifiers such as long vs. short, large vs. small, broad vs. narrow. Other examples given by Köhler et al. (2017) for either qualitative or quantitative characters (e.g., strongly pulsed vs. tonal calls, call series vs. single calls; duration of calls in seconds, dominant frequency in Hertz) could be also easily converted from one category into the other.

Operationally, a prompt construction of qualitative characters depends on the presence of natural gaps within character variation. In other words, attribute variation cannot be completely gradual. Instead, it must be clustered into two or more non-overlapping ranges. For example, if calls of different taxa have values of pulse number that are clustered into two clearly separated sequence groups, these calls could be classified into two categories or states for this character: few pulses vs. many pulses. Therefore, regarding character variation and polymorphism, the presence of natural gaps within a trait is usually what leads researchers to construct qualitative characters (Wiens 2000, see overlapping or disjunct characters in Thiele 1993). When gaps are clear (disjunct characters), features are described qualitatively because they are promptly organized into categories by the researcher and then described as such. On the other hand, when trait variation overlaps among taxa such that there is no gap, it is not as simple to delimit categorical states (cf. coding methods for continuous character in Wiens 2000) and traits are often described quantitatively (e.g., variation of note length in Köhler et al. 2017: 44). The key importance of gaps in relation to how traits are described (quantitatively vs. qualitatively) is recognized by Köhler et al. (2017: 47-48) where the authors state 'Such a difference [little or no overlap in their range values] in even a single call trait will potentially be as conclusive as a qualitative difference'. Since it is only an operational option to describe traits qualitatively or quantitatively, there cannot be any natural difference related to whether the characters are described numerically (quantitative) or in categories (qualitative). Instead, the reliability differences pointed by previous studies (e.g., Gerhard and Huber 2002, Köhler et al. 2017) seem to be related to whether the trait variations are overlapping or disjunct among taxa, 
which correspond to natural differences indeed (see Thiele 1993).

\section{NAMING BIOACOUSTICAL STRUCTURES}

In order to offer a practical alternative for naming bioacoustical structures centered on homology correspondences instead of universal similarities, we provide a guideline, step by step, focusing on first understanding the structures' features intraspecifically, then comparing them to the available variations in sister taxa, establishing homology, and finally choosing the best terms for these structures. For assessment of primary homology, we followed the correspondence criteria applied to behavioral expressions (Wenzel 1992), which were originally proposed by Remane (1952) for morphological structures. The so called Remane's criteria (see step five below) have been successfully applied specifically to acoustic emissions (e.g., Hepp et al. 2017, DesutterGrandcolas and Robillard 2003 and references within). The logic of the following steps aims to name any bioacoustical structure, from entire emissions to parts of them.

1) Obtain as many recordings as possible for the studied species, preferably including different individuals and populations in order to sample intraspecific variation. Such action reduces the chance of biased samples in species with widely varying acoustic emissions.

2) Delimit the observed structures and describe them as detailed as possible regarding to their spectral, temporal, and amplitude characteristics. If functions and/or producing mechanisms were accessed, they should also be described associated to the structures as any bioacoustical feature. At this moment, structures should be labeled with temporary symbols (e.g., alphabet letters or numbers).

3) Obtain signal recordings of as many phylogenetically closely related species as possible. This approach increases the chance of sampling intermediate forms that may connect properties of compared structures, reducing the risk of establishing homologous structures as nonhomologous by comparing a few forms significantly different from each other. Bioacoustical descriptions of closely related species in literature can be used in order to assess structure variations that have not been obtained through direct examination of audio recordings.

4) Examine the features of each structure of the closely related species. If necessary, take some of those measurements used to describe the structures of the species in focus (step two) and pay a special attention to the position in time of each structure in relation to the others within the emission (see next step). If there are call descriptions available that agree with the condition given in the step above, use the features and measurements presented in those.

5) Compare the structures of all species examined taking into account the bioacoustical features analyzed for the signals in the step two and four, including the relative position in time for each structure present in the emissions. Establish correspondences between the bioacoustical structures of the taxa studied based on similarities of their characteristics (I), similarity of their relative temporal position in the emission (II), and comparisons of the largest number of taxa available including as many intermediate forms as possible (III). This set of homology criteria is often called Remane's criteria (Remane 1952, Wenzel 1992) and each criterion is referred to as 'special quality' (I), 'position' (II), and 'connection by intermediates' (III) (Wenzel 1992). If other traits related to the signal, such as function, producing mechanism, motivation, direction, and number of airflows used to emit calls or structures within calls, 
are known, they should be taken into account as additional 'qualities' for the criterion (I). In cases of conflicting outcomes, those homology hypotheses based on more complex traits and/ or higher numbers of similarities should be favored (see A. Remane 1952, J. Remane 1983 and references within). Note that, although similarity is operationally used to connect structures, intermediate forms may connect structures that are not similar to each other but only to the intermediate ones. In this context, similarity is not a premise for structures to be homologous, although structures are connected by similarities.

6) Reassign the preliminary labels to the structures of the different species according to the correspondences. Use the same label to those structures considered homologous in step five. Repeat the procedure for all the bioacoustical structures examined until you have assigned a preliminary label to every described structure.

7) Finally, replace the preliminary labels with names that have been frequently used in the literature for the structures described in order to guarantee terminological stability over time. If there are two or more commonly used names for the same structure, we suggest that the definitions of the alternative terms are considered as a tiebreaking criterion. For these cases, the term with the definition that best fits the characteristics of the given structure should be used. It is important to note that the terminological predefinitions are used only as a way to choose the best term and do not interfere with the homology hypotheses.

By following this procedure, one will be able to apply a single and unique name for all structures considered homologous regardless of whether they are all similar or not (J. Remane 1983). We also suggest that all these steps are clearly given while describing the calls. This way, the propositions for the homology establishments (e.g., used criteria and supporting evidence) and terminology choices can be accessed and evaluated by subsequent studies. Moreover, clear homology hypotheses and the associated terminology should facilitate further applications by other researchers and consequently favor nomenclature stability.

\section{CONCLUSIONS}

Studies that present terminology options associated with clear definitions and possible variations for the acoustic structures and their properties are important to facilitate communication and, if well applied, can make bioacoustical descriptions clearer. For instance, by using the definitions based on general similarity such as those proposed by Toledo et al. (2015a) and Köhler et al. (2017), researchers will have a possibility to connect more objectively descriptions to bioacoustical features and structures (like a reference glossary; see steps three and seven in the guidelines above). However, researchers interested in the use of bioacoustical features in the field of comparative biology must keep in mind that homology assessment must be priority (Hall 2013) and that this assessment can be reached only by structure correspondence and not by obligatory similarity (Grant and Kluge 2004, Ghiselin 2005). Every attempt to standardize terms for acoustic structures by applying rigid definitions based on similarities, i.e., similarity-based diagnoses for structures, tends to lead to spurious homology assessments. The same homology criteria used to make correspondence among other phenotypical structures, such as morphological ones, can be used to bioacoustical structures (Desutter-Grandcolas and Robillard 2003 and references within). Usually, the successful use of these criteria depends on the comprehension degree of (I) the acoustic characteristics related to the structures in question (connection by special quality) and (II) those temporally close to them 
in the emission (connection by relative position), and on (III) the success of sampling intermediate forms (connection by intermediates; Remane 1952, Wenzel 1992). Regardless of the concern on homology assessment, we reinforce the importance of connecting the descriptive terms to the structures and properties, for instance, by labeling clearly the structures and traits with the used terms in illustrations, and making the recordings accessible to the public (see Toledo et al. 2015b). Finally, the application of the character categories 'qualitative' and 'quantitative' is occasional and has only operational utility. They do not refer to natural trait-properties (Thiele 1993). Instead, they only refer to traditions or momentary preferences of the researchers in describing characters and then no conclusion on differences of biological reliability between them makes sense.

\section{ACKNOWLEDGMENTS}

F.H. thanks J.M. Padial, M.A. Raposo, and K. Fitzhugh for having introduced him to the classical and modern philosophy of biological science; and J.W. Wenzel for insights on bioacoustical homology. We thank A.M. Bezerra, P.H. Pinna, and J.M. Padial for reviewing draft versions and for long-term discussions on the theme. We also thank the Conselho Nacional de Desenvolvimento Científico e Tecnológico (CNPq), Fundação Carlos Chagas Filho de Amparo à Pesquisa do Estado do Rio de Janeiro (FAPERJ), and Coordenação de Aperfeiçoamento de Pessoal de Nível Superior (CAPES) for financial support.

\section{AUTHOR CONTRIBUTIONS}

Both authors participated in elaboration of conceptualizing, theoretical analysis, discussion of results, and administration of the project. They also work together in the revision process and approval of final version of the manuscript. José
P. Pombal Jr. contributed in obtention of funds and supervision of the project.

\section{REFERENCES}

BOGERT CM. 1960. The influence of sound on the behavior of amphibians and reptiles. In: Lanyon WE and Tavolga WN (Eds), Animal sounds and communication, Washington, DC: AIBS, p. 137-320.

DE PINNA MGG. 1991. Concepts and tests of homology in the cladistic paradigm. Cladistics 7: 367-394.

DESUTTER-GRANDCOLAS L， LEGENDRE F, GRANDCOLAS P, ROBILLARD T AND MURIENNE J. 2005. Convergence and parallelism: is a new life ahead of old concepts? Cladistics 21(1): 51-61.

DESUTTER-GRANDCOLAS L AND ROBILLARD T. 2003. Phylogeny and the evolution of calling songs in Gryllus (Insecta, Orthoptera, Gryllidae). Zool Scr 32: 173-183.

DUELLMAN W AND TRUEB L. 1986. Biology of amphibians. New York: Mc Graw-Hill Inc, 610 p.

GERHARDT HC AND HUBER F. 2002. Acoustic Communication in Insects and Anurans: Common Problems and Diverse Solutions. Chicago and London: The University of Chicago Press, $531 \mathrm{p}$.

GHISELIN MT. 1974. A Radical Solution to the Species Problem. Syst Zool 23: 536-544.

GHISELIN MT. 2005. Homology as a relation of correspondence between parts of individuals. Theor Biosci 124: 91-103.

GRANT T AND KLUGE AG. 2004. Transformation series as an indeographic character concept. Cladisctics 20: 23-31.

HALL BK. 1994. Homology: The hierarchical basis of comparative biology. San Diego: Academic Press, 483 p.

HALL BK. 2013. Homology, homoplasy, novelty, and behavior. Dev Psychobiol 55: 4-12.

HENNING W. 1966. Phylogenetic systematics. University of Illinois Press, $130 \mathrm{p}$.

HEPP F, LOURENÇO ACC AND POMBAL JR JP. 2017. Bioacoustics of four Scinax species and a review of acoustic traits in the Scinax catharinae species group (Amphibia: Anura: Hylidae). Salamandra 53: 212-230.

KÖHLER J, JANSEN M, RODRÍGUEZ A, KOK PJR, TOLEDO LF, EMMRICH M, GLAW F, HADDAD CFB, RÖDEL MO AND VENCES M. 2017. The use of bioacoustics in anuran taxonomy: theory, terminology, methods and recommendations for best practice. Zootaxa 4251: 1-124.

MCLISTER JD, STEVENS ED AND BOGART JP. 1995. Comparative contractile dynamics of calling and locomotor muscles in three hylid frogs. J Exp Biol 198: 1527-1538.

REMANE A. 1952. Die Grundlagen des Natiirlichen Systems der Vergleichenden Anatomie und der Phylogenetik. Leipzig: Geest und Portig K.G., 400 p. 
REMANE J. 1983. The concept of homology in phylogenetic research - its meaning and possible applications. Paläont Z 57: 267-269.

RENDALL D AND DI FIORE A. 2007. Homoplasy, homology, and the perceived special status of behavior in evolution. J Hum Evol 52: 504-521.

ROBILLARD T AND DESUTTER-GRANDCOLAS L. 2011. The complex stridulatory behavior of the cricket Eneoptera guyanensis Chopard (Orthoptera: Grylloidea: Eneopterinae). J Insect Physiol 57(6): 694-703.

ROBILLARD T, HÖBEL G AND GERHARDT HC. 2006. Evolution of advertisement signal in North American hylid frogs: vocalizations as end products of calling behavior. Cladistics 22: 533-545.

STRIEDTER GF AND NORTHCUTT RG. 1991. Biological hierarquies and the concept of homology. Brain Behav Evolut 38: 177-189.

THIELE K. 1993. The Holy Grail of the perfect character: The cladistic treatment of morphometric data. Cladistics 9: 275-304.
TOLEDO LF, MARTINS IA, BRUSCHI DP, PASSOS MA, ALEXANDRE C AND HADDAD CFB. 2015a. The anuran calling repertoire in the light of social context. Acta Ecol 18: 87-99.

TOLEDO LF, TIPP C AND MÁRQUEZ R. 2015b. The value of audiovisual archives. Science 347: 484.

WELLS KD. 1977. The social behaviour of anuran amphibians. Anim Behav 25: 666-693.

WELLS KD. 2007. The ecology and behavior of amphibians. Chicago and London: The University of Chicago Press, 693 p.

WENZEL JW. 1992. Behavioral homology and phylogeny. Ann Rev Ecol Syst 23: 361-381.

WIENS JJ. 2000. Phylogenetic analysis of morphological data. Washington and London: Smithsonian Institution Press, $220 \mathrm{p}$.

WIENS JJ. 2001. Character analysis in morphological phylogenetics: problems and solutions. Syst Biol 50: 689699. 